\title{
EL SÍNDROME DE BURNOUT EN LOS PROFESIONALES DE ODONTOLOGÍA
}

\author{
Mariella Bazalar Herrera y Gunther Balarezo López
}

\section{Resumen}

El Síndrome de Burnout constituye uno de los daños laborales de carácter psicosocial más importantes en la sociedad actual. El ritmo de vida, la transformación de la estructura económica, las exigencias en el trabajo y los costos que este sindrome supone para las personas y las organizaciones, han despertado el interés en conocer y tomar medidas para prevenirlo. En el caso de la odontología, parece ser que son los profesionales de la salud con mayor prevalencia de este síndrome. Para ello, una detección temprana del problema puede ayudar a que no se vea afectada su salud y su vida familiar y laboral.

Palabras clave: sindrome de Burnout; estrés; odontología.

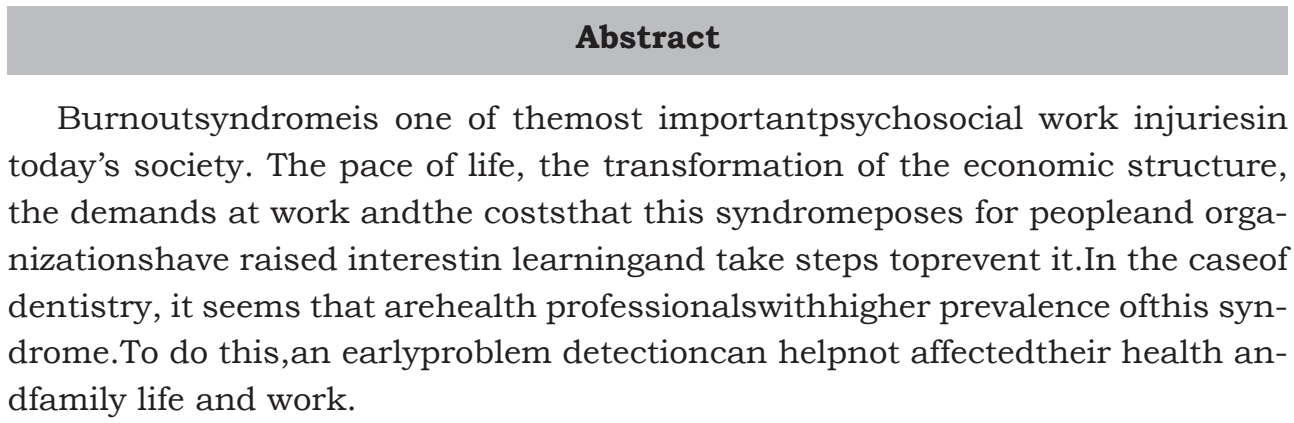

Key words: Burnout syndrome; stress; dentistry. 
El sindrome de Burnout, es un término anglosajón cuya traducción es estar desgastado o exhausto. Aunque no existe definición unánimemente aceptada, sí parece haber consenso en que se trata de una respuesta al estrés laboral crónico, lo que engloba implicaciones nocivas para la persona y la organización. En tal sentido, la presencia de este síndrome se considera una consecuencia de trabajar intensamente, de no tomar en cuenta las necesidades personales y se relaciona a sentimientos de falta de ayuda, de impotencia, de actitudes negativas y de una posible pérdida de autoestima. (Guerrero, 2015; Ríos, 2007)

Este término es descrito por primera vez por el psiquiatra Freudenberger en los años setenta,el cual prestaba sus servicios en una clínica para toxicómanos en Nueva York (EEUU). Él observó que después de un año de encontrarse laborando, la mayoría de los voluntarios sufrían al mismo tiempo una pérdida gradual de energía hasta llegar al agotamiento y mostrar sintomas de depresión y ansiedad. En la década de los ochenta, la psicóloga social Cristina Maslach, al estudiar las respuestas emocionales, denomina a estos sintomas "sobre carga emocional" o "sindrome de Burnout"; posteriormente, crea un instrumento de valoración, el Maslach Burnout Inventory (MBI), (Maslasch, 1986) que mide tres dimensiones: el cansancio emocional, la despersonalización y la realización personal. (Freudenberger, 1974; Maslach, 1986; Mingote, 2004; Acevedo, 2014).
Estrés y Burnout se han considerado por mucho tiempo términos que hacen referencia al mismo fenómeno, existiendo una confusión conceptual. Por un lado, el estrés se describe como una "respuesta física y emocional nociva que ocurre cuando los requerimientos del trabajo no son compatibles con las capacidades, los recursos o las necesidades de los trabajadores"; mientras que Burnout es básicamente un concepto que toma como base el hecho de que un síndrome es un conjunto de signos y sintomas (agotamiento, sintomas de ansiedad, depresión, desmotivación en el trabajo y agresividad hacia los demás), y aunque en el estrés hay también signos y síntomas, se considera que Burnout es un abordaje más completo del estrés crónico y se manifiesta como una consecuencia de este último. (Llaja, 2007)

En los inicios, se mencionaba que el síndrome de Burnout se podía dar como consecuencia de estar en contacto prolongado con personas que están en una situación de necesidad o dependencia. Más adelante, se propuso una definición más amplia y no restringida a las profesiones asistenciales, debido a que las excesivas demandas psicológicas no se dan únicamente en el servicio directo al público, sino que pueden darse también en otros ámbitos laborales, sobre todo en las que se mantiene un contacto continuo con personas (pacientes, clientes, alumnos, etc.), dado que las excesivas demandas imposibles de satisfacer, son las que producen la vivencia de fracaso personal. (Fidalgo, 2011; Escriba-Aguir, 2008). 
En tal sentido, las condiciones ocupacionales son consideradas estresantes para la mayoría de las personas, las cuales están asociadas a riesgos psicosociales, como trabajos muy demandantes o bajo presión, falta de control sobre las cargas y procesos de trabajo, carencia de apoyo social o de colegas y/o supervisores de trabajo, discriminación, aislamiento, acoso psicológico, falta de participación en la toma de decisiones, pobre comunicación o flujo de información, inseguridad laboral, ausencia de oportunidades de crecimiento, avance o promoción, horarios irregulares(turnos de trabajo) y estar expuesto a condiciones fisicas desagradables o peligrosas $\mathrm{y}$ no tener control sobre estas condiciones. (Houtman, 2011).

Asimismo, cabe mencionar que, aunque las características organizacionales e individuales juegan un papel importante en el desarrollo del estrés laboral, este tipo de estrés resulta de la interacción entre el trabajador y las condiciones de trabajo; las diferencias individuales del trabajador, tales como personalidad, edad, nivel educativo, experiencia y las formas de enfrentar el problema, la frecuencia de órdenes contradictorias de parte del jefe inmediato, no contar con apoyo informativo, emocional, instrumental de parte de los compañeros de trabajo $\mathrm{y}$ no realizar actividades recreativas, de esparcimiento o descanso en horas y dias libres fuera del hospital. Estas variables pueden ayudar a predecir si determinadas características del trabajo podrian incidir en el estrés.
(Houtman, 2011; Yslado, 2012).

Es por ello que el estrés ha pasado de ser un problema individual/personal, para convertirse en un problema laboral/social, porque los primeros sentimientos negativos que se detectan son contra los desencadenantes del proceso: jefes y usuarios, después los amigos, la familia $\mathrm{y}$, finalmente, la propia persona, lo que supone una pérdida de autoestima y depresión. (Medina, 2011; Sos Tena, 2002).

Según el Gerente de Desarrollo de Negocios de WHALECOM, Consultora Especializada en Procesos de Cambio, "En los últimos años, la disciplina del Estrés Management ha logrado varios avances en la identificación de las principales causas organizacionales de estrés laboral" como: (Alonso, 2011)

1. Cultura organizacional: las culturas organizacionales ultra competitivas suelen devenir en ambientes laborales tóxicos. La falta de apoyo de colegas y superiores es una causa frecuente de estrés. En casos extremos, estos ambientes pueden desencadenar en conductas de acoso laboral (mobbing).

2. Grado de exigencia: las personas con poco o demasiado trabajo, son más proclives a sufrir estrés.

3. Control sobre el trabajo: los empleados a quienes se otorga la posibilidad de elegir la manera de realizar su trabajo suelen presentar menos síntomas de estrés laboral.

4. Cambios organizacionales: los 
procesos de cambio organizacional son una importante causa de estrés. En este caso, las variables clave, son:¿Cuánta información reciben los empleados sobre el cambio? ¿El proyecto ha sido planificado o sorpresivo?

5. Función laboral y capacitación: los empleados que no tienen claro en qué consiste exactamente su trabajo y no reciben suficiente capacitación suelen presentar mayores índices de estrés.

Por otro lado, el estrés laboral también puede ser el resultado de un pobre balance en la relación hogar/ trabajo, con severas consecuencias cuando convergen la pobreza, el riesgo de desempleo y condiciones de vida precarias. Esto puede afectar principalmente a las mujeres en países con disparidades de género, quienes en los lugares de trabajo experimentan y expresan con mayor frecuencia que los hombres, problemas asociados al estrés, siendo las causas más frecuentes:

- doble rol que deben jugar tanto en el hogar como en el trabajo, y la dificultad para balancear estos roles;

- los roles sociales de género y la necesidad de un rol independiente contra la necesidad de conformarse a las expectativas sociales;

- el acoso sexual en el trabajo;

- la discriminación de género, que se traduce en menores ingresos y mayores exigencias y requisitos ocupacionales.

El síndrome de Burnout, es un problema de salud complejo, que no solo comprende las tres dimensiones señaladas, pues también genera problemas de salud, física, mental, afecta la calidad de prestación de servicios de salud, la calidad de vida familiar y la calidad de vida en general, de quien la padece. (Yslado, 2012)

\section{Signos y Sintomas del Sindrome de Burnout}

Se han identificado cuatro fases de la evolución del síndrome de Burnout, que son: (Garcia, 2007)

- Leve: los afectados presentan síntomas físicos, vagos e inespecíficos (cefaleas, dolores de espaldas, lumbalgias), y se vuelven poco operativos. Uno de los primeros sintomas que sirve de primer signo de alarma, es la dificultad para levantarse por la mañana o el cansancio patológico.

- Moderada: aparece insomnio, déficit en atención y concentración, tendencia a la automedicación. Este nivel presenta distanciamiento, irritabilidad, fatiga, aburrimiento, progresiva pérdida del idealismo que convierten al individuo en emocionalmente exhausto con sentimientos de frustración, incompetencia, culpa y autovaloración negativa.

- Grave: mayor ausentismo, aversión por la tarea, abuso de alco- 
hol y psicofármacos.

- Extrema: aislamiento, crisis existencial, depresión crónica y riesgo de suicidio.

Entre los síntomas más comunes asociados la sindrome de Burnout, algunos autores mencionan los siguientes: ${ }^{6,10,16,17,18}$

1. Psicosomáticos: fatiga crónica, mareos, dolores de cabeza frecuentes, dificultades para conciliar el sueño, insomnio, úlceras $\mathrm{u}$ otros desórdenes gastrointestinales, infecciones respiratorias, taquicardias, depresión, alergias, desórdenes alimenticios, pérdida de apetito y de peso, hipertensión, asma, dolores musculares, falta de deseo sexual, pérdida de ciclos menstruales, trastornos dermatológicos, tics, en la cavidad oral bruxismo, herpes, aftas, entre otras.

2. Mentales: sentimientos de vacío, agotamiento, fracaso, impotencia, baja autoestima $\mathrm{y}$ pobre realización personal. Es frecuente apreciar nerviosismo, inquietud, dificultad para la concentración y una baja tolerancia a la frustración, concomportamiento paranoides y/o agresivos hacia los pacientes, compañeros y la propia familia.

3. Conductuales: abuso de alcohol y drogas, aumento en el consumo de café y fármacos, aumento de conducta violenta, comportamiento de alto riesgo, suicidio.

4. Emocionales: distanciamiento afectivo, irritabilidad, recelos, incapacidad para concentrarse, baja autoestima y deseos de abandonar el trabajo, ideación suicida.

5. Defensivos: negación de las emociones, ironía, racionalización, desplazamiento de afectos

6. Desempeño laboral: ausentismos y accidentes laborales, conflictos laborales, toma de decisiones con eficacia disminuida.

7. Familiar: divorcio, conflictos interpersonales

Aparte de los síntomas que sufre el sujeto, este síndrome también va a afectar a la familia y la calidad del trabajo. El individuo se quejará continuamente, hablará reiteradamente de su trabajo y se transformará en un problema para la familia. No es raro que los lamentos y agresividad se dirijan a otra persona o al cónyuge, transformando esta última situación en un riesgo de divorcio. Es frecuente que los sentimientos negativos que se generan por Burnout, terminen afectando no sólo a las personas relacionadas con el trabajo, sino a quienes lo rodean. ${ }^{16}$

En el trabajo, el resultado más evidente será una disminución del rendimiento que incide en la motivación, afecta la convivencia con los compañeros, impide cualquier felicidad personal o satisfacción profesional, incrementa los errores, obstaculiza cualquier intento de reforma laboral, aumenta la frustración y predomina una actitud de no participación. Se cae en una rutina, lo que suele llevar a una menor atención de las necesidades humanas al mostrar poca sensibilidad 
hacia los sentimientos del usuario. Esta deshumanización en el trabajo puede traer como consecuencia el uso de medidas inapropiadas. En las fases precoces, el profesional se desconecta emocionalmente del trabajo, más adelante se desconecta fisicamente (llega tarde, falta con frecuencia); si la situación se mantiene, pronto pensará en cambiar de trabajo, donde tenga menos contacto con la gente. (Arauco, 2008)

El Sindrome de Burnout también da lugar a una disminución de la productividad; es decir, perjudica el desempeño laboral. Así mismo, es un importante mediador patogénico que daña la salud del profesional, tanto por los efectos directos que ocasiona, como por los indirectos, a través de adquisición de conductas nocivas para la salud, considerados estos como mecanismos de defensa (consumo de alcohol, drogas, tabaco, etc.). Es así que las tasas de alcoholismo y adicción a drogas, así como los conflictos matrimoniales, trastornos psiquiátricos, depresión y suicidio, son superiores en las personas que sufren de este sindrome que en el resto de la población. (Arauco, 2008)

En todos los casos, es un malestar general pero a menudo imposible de identificar; es como una mezcla de hastío, tristeza y desgano sin causa ni motivo preciso.

\section{Sindrome de Burnout y Odontologia}

La práctica dental ha sido considerada como la que cada vez más en la clínica presenta características y experimenta más problemas de salud físi- ca y mental, en comparación con otras profesiones sanitarias. Esto se debe porque ha sido reconocida como una actividad demandante. Para desempeñar sus funciones necesitan gran variedad de equipos y materiales, por lo que están expuestos a sufrir diversos riesgos de trabajo, como son accidentes y el estar en contacto con diversos agentes infecciosos; todo esto, aunado a las nuevas tecnologías utilizadas, cada vez más sofisticadas, que hacen que su labor profesional sea más complicada. (Castañeda, 2012)

Los factores que inciden en el aumento de la tensión en los odontólogos, son: el realizar su actividad laboral en un área limitada, con exigencias de tiempo entre un paciente y otro; las cuestiones ergonómicas, como bipedestación prolongada, posturas forzadas y restringidas exclusivamente a la cavidad bucal, en condiciones no óptimas, con procedimientos quirúrgicos y dentales prolongados; además de un ambiente de trabajo ruidoso, con insuficiente iluminación y ventilación, así como los factores ambientales que influyen negativamente en la productividad de ellos, una estructura organizacional y un ambiente laboral donde no existe la comunicación entre los mismos trabajadores. (Wycoff, 1984; Pollack, 1996, Freeman, 1995; Romero, 2001; Turley, 1993)

El trabajo del cirujano dentista en el ámbito profesional es arduo, tenso, e implica un deterioro físico y mental, ya que realiza su trabajo con apremio de tiempo, repetición de los tratamientos, ansiedades de los pacientes y los 
problemas a la hora de cobrar los honorarios, lo que contribuye a que sean los profesionales sanitarios más ansiosos. (Mondol, 2008)

En cuanto al ambiente laboral, este es una fuente de estrés para el odontólogo por la posibilidad de contagio de enfermedades, tales como Hepatitis B y Hepatitis C. Por ejemplo, los odontólogos poseen diez veces más riesgo de adquirir una Hepatitis B que el promedio de ciudadanos. El lugar de trabajo se asocia a la humedad y un alto riesgo de daño a la piel y las manos, por estar expuestos a sustancias irritantes de los materiales dentales y los guantes; a un ambiente ruidoso (con sus constantes cambios en la intensidad y la frecuencia de los sonidos); y al deslumbramiento de la lámpara; los olores de los materiales y desinfectantes, lo cual puede estimular los sentidos y afectar el humor y el estado mental. Además, se tiene que tratar con pacientes de diferente personalidad (hostiles, miedosos, agresivos, fóbicos e hipocondriacos). (Arauco, 2008; Feresin, 2011)

Asimismo, el odontólogo está sujeto a las expectativas y constante evaluación del paciente y al desgaste emocional que supone la interacción tratamiento-salud. Además, existen una serie de factores predisponentes que hacen que los profesionales de este campo sean más susceptibles al desgaste profesional, como son: (López, 2006; Barraza, 2006)

- Sobrecarga laboral: el tiempo para cada tratamiento está prefijado en un horario donde no se incluye la atención inmediata a urgencias, complicaciones del tratamiento y las consecuencias de la tensión emocional del paciente.

- Ejercicio solitario: la responsabilidad no puede ser compartida y es frecuente la sensación de soledad.

- Ambigüedad en la relación paciente-profesional: la desconfianza económica y el miedo al dolor se enfrentan con otros sentimientos, como necesidad y desamparo.

- Riesgo económico: siempre está presente al trabajar como autónomo y gestionar su propia empresa.

- Ejercicio profesional: en lugares cerrados y con ambiente contaminado.

De otro lado, que los profesionales en odontología tienen una alta prevalencia de enfermedades relacionadas con el síndrome de Burnout, ya que todas sus características son nombradas en algún grado, siendo las molestias musculo-esqueléticas las de mayor prevalencia, seguido de las psicosomáticas $y$, por último, las enfermedades gastrointestinales y cardiovasculares. ${ }^{16}$ Además, no existe diferencia entre los niveles de estrés reportados en diferentes especialidades odontológicas, aunque los odontopediátras muestran mayores porcentajes de estrés respecto a los demás. Se considera que los mayores índices de suicidios entre profesionales de la salud se da en odontólogos, psiquiatras 
y anestesiólogos y son ellos los que sienten mayor presión por la pérdida de perspectiva profesional y el mantenimiento del consultorio, mientras que las odontólogas sienten mayor presión por la incertidumbre de situaciones profesionales. (Feresin, 2011; Albanesi, 2006)

Finalmente, existe una obligación continua de estudio y puesta al día. Generalmente, los dentistas se informan de las nuevas tecnologías mediante conferencias y cursos que se imparten. Estos se han descrito como una fuente de ansiedad, porque el odontólogo se va a encontrar ante la dicotomía de asistir o no. Por un lado, se puede interpretar como un aumento de conocimientos, pero, por otro lado, necesita encontrar tiempo o un reemplazo en la consulta para poder asistir. A ello se suma el aspecto económico, debido al gasto que debe asumir como derecho a asistir y que sus ingresos se pueden ver afectados por las horas que dejará de atender en el consultorio. (Mondol, 2007; Bedoya, 2006)

Por todo lo mencionado, a continuación se dan algunas recomendaciones para la prevención del síndrome de Burnout en los odontólogos: (D' Angelo, 2010)

- elija un campo de trabajo y asegúrese una formación e información suficiente. Esto disminuye la angustia por los imprevistos y los malos resultados;

- establezca un ritmo de trabajo que sea llevadero para usted;

- cultive una buena comunicación con sus colegas, en su lu- gar de trabajo y/o fuera de él;

- esté alerta para tomar en serio todos los indicios de estrés apenas comience a manifestarse;

- identifique claramente estos indicios, y converse con personas que puedan reflexionar con usted, a fin de comprender $y$ modificar las situaciones y los contextos en el que el estrés se reitera;

- si no puede cambiar el marco de su trabajo, es necesario que cambie usted;

- asuma el compromiso y la prioridad de realizar actividades para la salud, fisica y mental en forma periódica y continuada. Elija algo que verdaderamente le guste;

- aprenda a encarar y resolver sus conflictos familiares y personales. Nada hay más estresante que tener miedo de volver a casa.

\section{Conclusiones}

La prevención y tratamiento del Sindrome de Burnout requiere un enfoque multidisciplinario. La etiología de dicho sindromes se encuentra relacionado a factores individuales, organizacionales y socio-culturales. El hecho de percatarse a tiempo y reconocer el problema constituye un elemento determinante para la pronta y efectiva resolución del mismo.

En el caso de los odontólogos, se describen como perfeccionistas y competitivos, y para ellos es esencial man- 
tener estándares altos para conservar el nivel de calidad de su trabajo. Pero el perfeccionismo, caracterizado por demandas criticas y constantes, va más allá del deseo de dar lo mejor de sí mismo y es frustrante cuando el perfeccionismo nunca se satisface. En un mundo obviamente imperfecto, las demandas de perfección inevitablemente son decepcionantes, y cuando no se cambia el perfeccionismo se puede volver un estresor crónico. Por ello, Forrest menciona que "la búsqueda del perfeccionismo es una de las mayores causas de estrés y frustración en la Odontologia".

Se hace evidente que el bienestar o la salud total no podrá conseguirse en el futuro solo con medicinas. Será necesario avanzar también en otras áreas, como educación, trabajo y seguridad, como requisitos de la promoción y prevención en salud. Por ello, se hace necesario estudiar el síndrome de Burnout, porque está asociado al derecho a la protección de la salud que toda persona tiene en cuanto está insertado en una organización, ya que produce daño por causas de exposición a riesgos psicosociales y organizativos. (Moreno, 2001)

Finalmente, en la literatura actual se menciona otra forma de hacer frente a este sindrome, y es el término "resiliencia", que significa la capacidad de asumir con flexibilidad situaciones limite y sobreponerse a ellas. Es un constructo multidimensional que facilita la superación de las adversidades gracias a la capacidad de recuperarse y salir fortalecido y exitoso, pese a estar expuesto a un estrés psicosocial marcado. ${ }^{35}$ Se trata de una cualidad dinámica que se encuentra latente en cada persona y que permite enfrentar los estresores laborales de hoy en día. El punto crucial es comprender que el estrés laboral no es por una debilidad individual del empleado; por el contrario, es un sintoma de que algo no marcha bien en la empresa. 


\section{REFERENCIAS BIBLIOGRÁFICAS}

Guerrero E. (2015). Una investigación con docentes universitarios sobre el afrontamiento del estrés laboral y el sindrome del quemado. [Citado 2015 ene 3]. Disponible en: www.rieoei.org/deloslectores/052Barona.PDF

Ríos B. Acuña R. (2007). Prevalencia y factores asociados en profesionales serums de la red de servicios salud de Huamanga, Ayacucho. Universidad Peruana Unión, Lima.

Freudenberger HG. (1974). Staff Burn-out. J Soc Issues;30:159-65.

Maslach C, Jackson SE. (1986). Maslach Burn-out Inventory. Palo Alto: Consulting Pysichologists Press.

Mingote Adan J, Moreno Jiménez B, Gálvez Herrer M. (2004). Desgaste profesional y salud de los profesionales médicos: revisión y propuesta de prevención. MedClin; 123:265-70.

Acevedo Figueroa L. (2014). Síndrome de Burnout en el profesional de enfermeria. Tesis para optar el Grado de Maestra en Ciencias de Enfermeria, Facultad de Enfermería, Universidad Autónoma de Querétaro, Santiago de Querétaro.

Llaja V. Sarriá C. García P.(2007) Síndrome del "Quemado" por estrés laboral asistencial, Manual, 1ra Edición, Lima.

Fidalgo M. (2011) Sindrome de estar Quemado: definición, proceso y degeneración.[citado 2011 ene 3]. Disponible en: www.insht.es/InshtWeb/Contenidos/Documentacion/.../NTP/.../ntp 704.pdf

Escriba-Aguir V, Artazcoz L, Pérez-Hoyos S. (2008). Efecto del ambiente psicosocial y de la satisfacción laboral en el sindrome de burnout en médicos especialistas.GacSanit 2008;22(4):300-308.

Houtman I. Jettinghoff K. (2011). Sensibilizando sobre el estrés laboral en los países en desarrollo. [Citado 2011 ene 13]. Disponible en: whqlibdoc.who.int/ publications/2008/9789243591650_spa.pdf

Yslado M. R, Atoche B. Rosa, Cermeño B. D, Rodríguez A. D, Sáchez B. J. (2013). Síndrome de Burnout y factores sociodemográficos--organizativos en profesionales de salud. hospitales del Callejón de Conchucos, Ancash, Perú - 2012. Universidad Nacional Antúnez de Mayolo Huaraz, Revista IIPSI 2013;16(1):191209.

Medina S. (2011). El estrés laboral organizacional asociado al bruxismo en trabajadores de la zona metropolitana de Guadalajara. [Citado 2011 ene 9]. Disponible en: cvsp.cucs.udg.mx/drupal6/documentos/estreslaboral.pdf

Sos Tena P, Sobrequés Soriano J, Segura Bernal J, et al. (2002). Desgaste profesional en los médicos de atención primaria de Barcelona. Medifam 2002;12(10):17-25.

Alonso M. (2011). Prevención y Detección del Síndrome de Burnout en los profesionales sanitarios. [Citado 2011 ene 18] Disponible en: www.fundacionprevent.com/Servicios/.../nextgrup_junio08.pdf 
García J. (2007). Estudio sobre variables de personalidad y organizacionales que influyen en el sindrome de burnout en los trabajadores de un hospital psiquiátrico penitenciario, Tesis para optar el Grado de Doctor, Departamento de Personalidad, Evaluación y Tratamientos Psicológicos, Facultad de Psicología, Universidad de Sevilla, Sevilla.

Arauco Pérez GV, Durand Barreto J. (2008). Síndrome de Burnout en Enfermeras del Hospital Nacional Especializado "Víctor Larco Herrera" 2007. Universidad Nacional Mayor de San Marcos, Lima.

D’angelo P. (2010) Diagnóstico, Prevención y tratamiento del estrés profesional y el Burnout del odontólogo. [Citado 2010 ago 20].Disponible en: www. patriciadangelo.com.ar/.../stressyburnout.htm

Estrés laboral. [Citado 2011 ene 13]. Disponible en: www.fade.es/ prevencion/22estres.pdf

Castañeda Aguilera E, García de Alba García JE. (2012). Prevalencia del síndrome de desgaste profesional (burnout) en odontólogos mexicanos del sector educativo y privado. Med. segur. trab. 2012;58(228):246-260.

Wycoff S. (1984). An examination of what dentists already know about stress and burnout within Dentistry. CDA Journal 1984; 12 (12): 114-117.

Pollack R. (1996). Dental office ergonomics: How reduce stress factors and increase efficiency. J Can Dent Assoc; 62 (6): 508-510.

Freeman R, Main J, Burke F. (1995). Occupational stress and Dentistry: theory and practice Part I Recognition. Brit Dent J 1995; 178 (6): 214-217

Romero R, Becerra T, Velasco M. (2001) Sindrome de Burnout. Desgaste emocional en cirujanos dentistas. Revista ADM 2001; 58 (2): 63-67.

Turley Kinirons M, Freeman R. (1993). Occupational stress factors in hospital dentists. Brit Dent J 1993; 175 (8): 285-288.

Mondol López M. (2008). Análisis de la prevalencia del Síndrome de Burnout en los odontólogos y sus repercusiones en la salud del profesional y en la atención bucodental del servicio de odontología en la Clínica Carlos Durán durante el periodo Agosto-Noviembre 2007. Tesis para optar el grado de Maestría, Instituto Centroamericano de Administración Pública, San José, Costa Rica.

Feresín C. (2011). Nuevas consideraciones sobre Burnout o Sindrome de Quemarse en el Trabajo (SQT) [Citado 2011 ene 27] Disponible en:www.psi.uba. ar/.../psicologia/...trabajo1/cdcongreso/.../

Efectos de la Globalización en el ámbito laboral de la atención de la salud de sus trabajadores (2005). [ Citado 2010 ago08] Disponible en:www.opas.org.br/ gentequefazsaude/bvsde/bvsacd/cd49/ambito.pdf

López M, Marín D, Campillo J. (2004). El Síndrome de Burnout y su incidencia en odontopediátras. Rev odontológica de Odontopediatría 2004;12(2);63-71.

Barraza A. (2006). Un modelo conceptual para el estudio del estrés académico. Rev. de psicología Iztacala 2006;9(3): 
Odontología y estrés. [Citado 2010 ago 15]). Disponible en: www.medilegis. com/.../Odontologica...ejercicio/ejercicio.htm

Albanesi S,Tifner S,Nasetta J. (2006). Estrés en odontología. Acta odontol. venez. 2006;44(3):310-315.

Gorter R, Eijkman M, Brake JK. (2001). Stress and health in dentists. Ned. Tijdschr. Tandheelkd 2001;108(2):54-58.

Bedoya L,Aranceli S, Perea M,Ormeño R. (2006). Evaluación de niveles, situaciones generadoras y manifestaciones de estrés académico en alumnos de tercer y cuarto año de una Facultad deEstomatología.Rev. Estomatol. Herediana 2006;16(1):15-20.

Moreno E. (2001). La Salud Pública en el Siglo XXI.Rev de Salud Pública Salud PublicaEduc Salud 2001;1(1):12-18.

Real Academia Española. [Citado 2015abr 15]).

Disponible en:http://lema.rae.es/drae/?val=resilencia 